\title{
ON A CERTAIN CLASS OF CANONICAL FORMS.*
}

BY MR. RALPH A. ROBERTS.

AN interesting class of theorems occurs occasionally in the consideration of algebraical quantics, viz., when a quantic (or quantics) is not in general reducible to a form (or forms) which at first sight, when we count the number of constants involved, appears to be sufficiently general to admit of a finite number of reductions. Such cases bear an analogy to the porism in geometry, as the reductions are impossible, except when the quantic (or quantics) satisfies an invariant relation, and then the number of reductions is infinite. These cases are not common in binary quantics, and are not very remarkable when they do occur. An instance is: Let $x_{1}, x_{2}, x_{3}$ be three linear expressions in the variables. Then $x_{1} x_{2} x_{3}$ and $a x_{1}{ }^{3}+b x_{2}{ }^{3}+c x_{8}{ }^{3}+d x_{1} x_{2} x_{3}$ contain six constants, viz., three involved in $x_{1}, x_{2}, x_{3}$ and the three external constants $a / b / c / d$; but two general binary cubics which contain six constants cannot be simultaneously reduced to these forms, unless the combinantive invariant obtained by substituting differential symbols in one and operating on the other vanishes. This, however, is readily apparent from the fact that there is an jdentical linear relation connecting the three cubes $x_{1}{ }^{3}, x_{2}{ }^{3}, x_{3}{ }^{3}$ and the product $x_{1} x_{2} x_{3}$, so that the second form is less general than it appears to be at first sight.

But in ternary and quaternary quantics there are several striking cases. The most remarkable, perhaps, is that discovered by Lüroth, viz, that a general plane quartic curve cannot be expressed linearly in terms of the fourth powers of five lines; say

$$
a_{1} x_{1}^{4}+a_{2} x_{2}{ }^{4}+a_{3} x_{3}{ }^{4}+a_{4} x_{4}{ }^{4}+a_{6} x_{5}{ }^{4} .
$$

I insert here a proof of this result, as it involves a method which I propose to use farther on.

Consider the unique conic $\Sigma$ which can be described to touch the five lines. Then substituting differential symbols, i.e., $\frac{d}{d x}, \frac{d}{d y}, \frac{d}{d z}$, for $\lambda, \mu, v$ in the tangential equation $(A, B, C, \ddot{F}, G, H)(\lambda, \mu, v)^{2}$ of $\Sigma$, and operating with the resulting expression on (1), the remainder vanishes identically. For, operating with $(A, B, C, F, G, H)\left(\frac{d}{d x}, \frac{d}{d y}, \frac{d}{d z}\right)^{2}$ on $x_{1}{ }^{4}=\left(\alpha_{1} x+\beta_{1} y+\gamma_{1} z\right)^{4}$, we get a result proportional to $\left(A \alpha_{1}^{2}+B \beta_{1}^{2}+C \gamma_{1}^{2}+2 F \beta_{1} \gamma_{1}+2 G \gamma_{1} \alpha_{1}+2 H \alpha_{1} \beta_{1}\right) x_{1}^{2} ;$ and this vanishes because $x_{1}$ touches $\Sigma$.

\footnotetext{
* Read before the American Mathematical Society, December 28, 1894.
} 
But with the quartic in a general form, if we operate with the result of substituting differential symbols in the tangential equation of a conic we obtain another conic in its direct form, and in order that this should vanish identically the six coefficients must separately be equal to zero. Thus we can eliminate the six constants in $\boldsymbol{\Sigma}$ and obtain an invariant relation. The latter is in fact, the determinant formed by the six second differential coefficients of the quartic. (See Salmon's Higher Plane Curves.)

Another form mentioned by Salmon is

$$
A_{1} x_{2} x_{8} x_{4} x_{6}+\text { etc. }+A_{8} x_{1} x_{2} x_{8} x_{4},
$$

to which a general quartic cannot be reduced.

In this case the proof follows from the fact that this form is a covariant of (1), and thus cannot contain any more constants than the latter does. But another proof can be found by a method given by Darboux in his work "Sur une classe remarquable de courbes et de surfaces algébriques" (Paris, 1873), viz., by using as co-ordinates the parameters of the two tangents which can be drawn from a point to the conic touching the five lines. It will then be seen that the form (2) is one of an infinite number of the same kind.

It may be worth while observing that two cubic curves cannot be written in the forms

$$
\left.\begin{array}{l}
\alpha_{1} x_{1}^{3}+\alpha_{2} x_{3}^{3}+\alpha_{8} x_{3}^{3}+\alpha_{4} x_{4}^{3}+\alpha_{8} x_{6}^{3} \\
\beta_{2} x_{1}^{3}+\beta_{2} x_{3}^{3}+\beta_{8} x_{3}^{3}+\beta_{4} x_{4}^{3}+\beta_{5} x_{5},
\end{array}\right\}
$$

although they appear to contain 8 constants externally and 10 constants in the lines; that is, 18 constants altogether, which should be exactly right for two cubic curves with 9 constants each. The proof easily follows as before, and the determinant formed by the six first differential coefficients is found to vanish.

Coming now to three dimensions, I observe that a remarkable case is given in Salmon's Surfaces. It is shown there that three quadrics cannot in general be expressed linearly in terms of the squares of five planes, although it would appear that there were a sufficient number of constants; viz., 12 constants externally and 15 implicitly in the five planes, that is, 27 altogether, which should be sufficient for three quadrics with nine constants each. It would also appear that a quaternary quartic could not be expressed as the sum of nine fourth powers, although such a form would appear to contain 35 constants-more than sufficient to express a general quaternary quartic, which contains 34 constants. The proof can be obtained by considering the unique quadric touching the nine 
planes; and the determinant formed by the ten second differential coefficients is found to vanish. I am not aware whether this result, as well as that relating to the two plane cubics, has been noticed before.

I now proceed to consider a result I have arrived at of the kind explained above; but before doing so I propose to consider a reduction which leads up to my theorem, as it throws light upon the processes employed in the more general case.

To reduce a conic and a plane cubic curve to the forms

$$
\left.\begin{array}{l}
\alpha_{1} x_{1}^{3}+\alpha_{2} x_{2}^{3}+\alpha_{3} x_{3}^{3}+\alpha_{4} x_{4}^{3}, \\
\left.a_{1} x_{1}^{2}+\alpha_{2} x_{2}^{2}+\alpha_{3} x_{3}^{2}+\alpha_{4} x_{4}^{2} \cdot\right\}
\end{array}\right\}
$$

Counting the constants, we have 6 externally and 8 implicitly; that is, 14 altogether, which are exactly right for a cubic and. a conic, with nine and five constants, respectively.

Now consider a conic $\Sigma$ touching the four lines $x_{1}, x_{2}, x_{3}, x_{4}$; then if we substitute differential symbols in the tangential equation of $\Sigma$ and operate on the cubic and the conic, both the results will vanish identically, exactly in the way $I$ have explained before; for the result of the operation on the power of any line, $L^{n}$ say, is proportional to $L^{n-2}$ multiplied by the condition that $L$ should touch the conic.

But suppose we write the cubic in one of its canonical forms, i.e.,

$$
x^{3}+y^{3}+z^{3}+6 m x y z=0 ;
$$

and that the conic at the same time is $(a, b, c, f, g, h)(x, y, z)^{2}$ $=0$ : then, operating with the result of substituting differential symbols in the tangential equation of the other conic $\Sigma$ $(A, B, C, F, G, H)(\lambda, \mu, v)^{2}$ which touches the four lines $x_{1}, x_{2}, x_{3}, x_{4}$ in (4), we get

and

$$
(A+2 m F) x+(B+2 m G) y+(C+2 m H) z,
$$

and in order that these expressions should vanish identically, we must have, from the first,

$$
A+2 m F e=0, \quad B+2 m G=0, \quad C+2 m H=0 ;
$$

thus $\Sigma$ must be

$$
A\left(m \lambda^{2}-\mu \nu\right)+B\left(m \mu^{2}-v \lambda\right)+C\left(m \nu^{2}-\lambda \mu\right)=0,
$$

subject to the condition

$$
A(m a-f)+B(m b-g)+C(m c-h)=0 ;
$$


that is, $\Sigma$ must touch the four lines determined by the equations

$$
\frac{m \lambda^{2}-\mu \nu}{m a-f}=\frac{m \mu^{2}-\nu \lambda}{m b-g}=\frac{m \nu^{2}-\lambda \mu}{m c-h} .
$$

Hence it is plain that these four lines are the lines $x_{1}, x_{2}, x_{3}, x_{4}$ in (4), and that the reduction is unique, as it has appeared that there is only one system of such lines. If it is required to find the constants $\alpha_{1}$, etc., $a_{1}$, etc., in the forms (4), there is no difficulty in finding equations to determine these quantities.

Now this result in a plane suggests an extension to three dimensions. Suppose we wish to reduce a quaternary cubic and a quadric to the forms

$$
\left.\begin{array}{l}
\alpha_{1} x_{1}^{3}+\alpha_{2} x_{2}^{3}+\alpha_{3} x_{3}^{3}+\alpha_{4} x_{4}^{3}+\alpha_{6} x_{5}^{3}+\alpha_{8} x_{8}^{3}, \\
a_{1} x_{1}^{2}+a_{2} x_{2}^{2}+a_{3} x_{3}^{2}+a_{4} x_{4}^{2}+a_{6} x_{6}^{2}+a_{6} x_{6}^{2},
\end{array}\right\}
$$

respectively. Counting the constants, we have 10 externally and 18 implicitly in the 6 planes; that is, 28 altogether, which are exactly right for a quaternary cubic with 19 constants and a quadric with 9 constants. In proceeding to consider the reduction in this case, the extension is from a conic in the plane to a twisted cubic curve in space. Let us consider a twisted cubic osculating the six planes in (5). This is a definite unique curve, for the problem is precisely the same, though in the tangential sense, as to describe a twisted cubic passing through six points; and the latter admits of a single solution (see Salmon's Surfaces). Now (see loc. cit.) there are three quadric surfaces touching all the osculating planes of the twisted cubic, which are not connected by a linear relation, precisely as there are three quadric surfaces, not connected by a linear relation, which can be described to pass through the curve. Let $\Sigma_{1}, \Sigma_{2}, \Sigma_{3}$ be the tangential equations of these three quadrics: then from the forms (5) we see that the results of substituting differential symbols $\left(\frac{d}{d x}, \frac{d}{d y}, \frac{d}{d z}, \frac{d}{d u}\right)$ for the tangential co-ordinates in any one of the quadrics, or the quadric $l \Sigma_{1}+m \Sigma_{2}+n \Sigma_{3}$, and operat$i_{n}$ on the cubic surface and quadric vanish identically; for the result of substituting differential symbols in the tangential equation of a quadric and operating on the power of a plane, $L^{n}$ say, is proportional to $L^{n-2}$ multiplied by the condition that $L$ should touch the quadric.

Now, if we consider the cubic surface in the general or any other form, the result of substituting differential symbols in the tangential equation of a quadric and operating on the 
cubic will be of the form $A x+B y+C z+D u$, and in order that this should vanish identically we should have $A=0, B=0$, $C=0, D=0$; that is, four conditions. Consequently, from the three non-linearly connected quadrics touching the osculating planes of the twisted cubic curve, we should have, apparently, twelve conditions connecting the curve with the surface, that is, sufficient conditions to determine the curve completely if the surface were given. Then since the curve also should satisfy three conditions with the quadric, it might at a rough inspection appear that three invariant conditions should connect the quadric with the cubic. Such, however, is not the case. The apparent twelve conditions satisfied by the twisted cubic curve with the cubic surface are in reality only equivalent to ten, as I proceed to show.

Let the lines of reference be chosen so that the cubic curve may be represented tangentially by the equations

$$
\beta^{2}-\gamma \alpha=0, \quad \alpha \delta-\beta \gamma=0, \quad \gamma^{2}-\beta \delta=0,
$$

and let the canonical planes of the cubic surface, according to Sylvester's unique canonical form, be

$$
\alpha_{i} x+\beta_{i} y+\gamma_{i} z+\delta_{i}^{\prime} u=0,
$$

so that the cubic surface itself is

$$
\Sigma_{6}{ }^{1}\left(\alpha_{i} x+\beta_{i} y+\gamma_{i} z+\delta_{i} u\right)^{3}=0 .
$$

Then, $U$ being one of the three expressions in (6), if we operate with the result of substituting differential symbols in $U$ on the cubic surface, and express that the result vanishes identically, we get

$$
\Sigma \alpha_{i} U_{i}=0, \quad \Sigma \beta_{i} U_{i}=0, \quad \Sigma \gamma_{i} U_{i}=0, \quad \Sigma \delta_{i} U_{i}=0,
$$

which, on account of the three values of $U$, apparently gives twelve conditions. This is, however, not the case, in consequence of the two identical relations,

$$
\left.\begin{array}{l}
\delta\left(\beta^{2}-\gamma \alpha\right)+\gamma(\alpha \delta-\beta \gamma)+\beta\left(\gamma^{2}-\beta \delta\right)=0 ; \\
\gamma\left(\beta^{2}-\gamma \alpha\right)+\beta(\alpha \delta-\beta \gamma)+\alpha\left(\gamma^{2}-\beta \delta\right)=0 .
\end{array}\right\}
$$

Hence there remain ten conditions; and when these are satisfied I propose to show that the five canonical planes are osculating planes of the twisted cubic. Solving the equations (7), we have

$$
\frac{U_{1}}{\Delta_{1}}=\frac{U_{2}}{\Delta_{2}}=\text { etc. }=\frac{U_{\mathrm{b}}}{\Delta_{1}},
$$


where $\Delta_{1}, \Delta_{2}$, etc., are the determinants formed by the coefficients of the five planes. Hence the ratios $U_{1} / U_{2}$, etc., must have the same values, no matter which of the quadrics $U$ we select; that is,

$$
\frac{\beta_{1}^{2}-\gamma_{1} \alpha_{1}}{\beta_{2}^{2}-\gamma_{2} \alpha_{2}}=\frac{\alpha_{1} \delta_{1}-\beta_{1} \gamma_{1}}{\alpha_{2} \delta_{2}-\beta_{2} \gamma_{2}}=\frac{\gamma_{1}^{2}-\beta_{1} \delta_{1}}{\gamma_{2}^{2}-\beta_{2} \delta_{2}}, \text { etc. }
$$

Hence

$$
\frac{\beta_{1}^{2}-\gamma_{1} \alpha_{1}}{\alpha_{1} \delta_{1}-\beta_{1} \gamma_{1}}=\frac{\beta_{2}^{2}-\gamma_{2} \alpha_{2}}{\alpha_{2} \delta_{2}-\beta_{2} \gamma_{2}} \text {, etc. }
$$

From which it follows that all the five planes must satisfy equations of the form

$$
\beta^{2}-\gamma \alpha-k(\alpha \delta-\beta \gamma)=0, \quad \gamma^{2}-\beta \delta-k^{\prime}(\alpha \delta-\beta \gamma)=0,
$$

where $k, k^{\prime}$ are constants. Hence from the identities (8), if $\beta^{2}-\gamma \alpha, \alpha \delta-\beta \gamma$ and $\gamma^{2}-\beta \delta$ do not all vanish, we should have

$$
\begin{aligned}
& k \delta+\gamma+k^{\prime} \delta=0 \\
& k \gamma+\beta+k^{\prime} \alpha=0 .
\end{aligned}
$$

But if such equations were satisfied by the planes, they should all have a line in common, which result is contrary to hypothesis; therefore $\beta^{2}-\gamma \alpha, \alpha \delta-\beta \gamma$, and $\gamma^{2}-\beta \delta$ must all vanish for each of the planes; that is, the planes must be osculating planes of the twisted cubic. Hence the latter curve having five given osculating planes satisfies ten conditions, and therefore has still two degrees of freedom. But operating with the results of substituting differential symbols in the three tangential quadrics on the given quadric we get three conditions, which are one greater than the degrees of freedom. Thus it would appear that one relation must exist between the cubic surface and the quadric. In other words, the cubic surface and the quadric cannot be reduced to the forms (5), unless a certain invariant relation between them is satisfied. I proceed to find this relation in the case when the cubic surface and quadric are written in the forms

$$
\left.\begin{array}{l}
A x^{3}+B y^{3}+C z^{3}+D u^{3}+E v^{3}=0 \\
(a, b, c, d, f, g, h, l, m, n)(x, y, z, u)^{2}=0,
\end{array}\right\}
$$

where $x+y+z+u+v=0$, identically.

Now it can easily be verified that a twisted cubic curve osculated by the five planes, $x, y, z, u$, $v$, can be represented parametrically thus:

$$
\lambda: \mu: \nu: \beta=(t-\alpha)^{-1}:(t-\beta)^{-1}:(t-\gamma)^{-1}:(t-\delta)^{-1} \text {, }
$$


where $\lambda, \mu, \nu, \rho$ are tangential co-ordinates. The ratios of the differences of $\alpha, \beta, \gamma, \delta$ give the two degrees of freedom which the curve still possesses. Now by eliminating $t$ we may write down

$$
\left.\begin{array}{l}
(\beta-\gamma) \mu \nu+(\gamma-\alpha) \nu \lambda+(\alpha-\beta) \lambda \mu=0, \\
\beta-\delta) \mu \rho+(\delta-\alpha) \rho \lambda+(\alpha-\beta) \lambda \mu=0, \\
(\gamma-\delta) \nu \rho+(\delta-\beta) \rho \mu+(\beta-\gamma) \mu \nu=0,
\end{array}\right\}
$$

which are three tangential quadrics touching the osculating planes of the curve and not connected by a linear relation. Substituting, then, $\frac{d}{d x}, \frac{d}{d y}, \frac{d}{d z}, \frac{d}{d u}$ for $\lambda, \mu, \nu, \rho$, respectively, in these three expressions and operating on the quadric given above, we get

$$
\begin{aligned}
& (\beta-\gamma) f+(\gamma-\alpha) g+(\alpha-\beta) h=0 \\
& (\beta-\delta) m+(\delta-\alpha) l+(\alpha-\beta) h=0 \\
& (\gamma-\delta) n+(\delta-\beta) m+(\beta-\gamma) f=0
\end{aligned}
$$

whence

$$
\begin{aligned}
(h-l)(g-f)(f-m) & +(h-g)(m-h)(n-f) \\
& +(h-f)(h-l)(n-f)=0,
\end{aligned}
$$

which is consequently the invariant relation connecting the cubic surface and the quadric when they are capable of being written in the forms (5). Thus it appears that a cubic surface and a quadric cannot, in general, be reduced to the forms (5), and that when they are reducible to these forms, the reduction can take place in a singly infinite number of ways, all the planes $x_{1}, x_{2}$, etc., involved being osculating planes of a given twisted cubic.

\section{HAYWARD'S VECTOR ALGEBRA.}

The Algebra of Coplanar Vectors and Trigonometry. By R. BaLdWIN HaYward. Macmillan \& Co., 1892. 8vo, pp. xxix +343 .

Ir is a curious fact that while the English are the one nation which in elementary geometry clings to Euclid, the prototype of mathematical rigor, not only is most recent English mathematical work, however excellent in many respects, decidedly lacking in rigor of form, but many English mathematical writers of the present day show an entire lack of critical sense which if shown in elementary geometry would discredit a schoolboy. 